\title{
Heat-pulse propagation along nonequilibrium nanowires in thermomass theory
}

\author{
Antonio Sellitto $^{1 *}$, Patrizia Rogolino ${ }^{2}$, Isabella Carlomagno ${ }^{3}$ \\ ${ }^{1}$ Department of Industrial Engineering, \\ University of Salerno, Fisciano, Italy \\ ${ }^{2}$ Department of Mathematics and Computer Science, \\ University of Messina, Messina, Italy \\ ${ }^{3}$ Department of Mathematics, Computer Science and Economics, \\ University of Basilicata, Potenza, Italy \\ *Email address for correspondence: ant.sellitto@gmail.com \\ Communicated by David Jou and Vito Antonio Cimmelli \\ Received on December 16, 2014. Accepted on March 9, 2015.
}

\begin{abstract}
We analyze the consequences of the nonlinear terms in the heat-transport equation of the thermomass theory on heat pulses propagating in a nanowire in nonequilibrium situations. As a consequence of the temperature dependence of the speeds of propagation, in temperature ranges wherein the specific heat shows negligible variations, heat pulses will shrink (or extend) spatially, and will increase (or decrease) their average temperature when propagating along a temperature gradient. A comparison with the results predicted by a different theoretical proposal on the shape of a propagating heat pulse is made, too.

Keywords: heat-waves propagation; nonlinear effects; energy-pulse propagation; thermomass theory

AMS subject classification: $80 \mathrm{~A} 20,74 \mathrm{~J} 30$
\end{abstract}

\section{Introduction}

Like biotechnology and information technology, nanotechnology is a growing industry with the potential to greatly change the world in which we live. Engineering of nanosystems rapidly developed in recent years, and it currently allows to design and develop mechanical, optical and electronic devices, the characteristic sizes of which may be of the order of tens nanometers. Nowadays, nanotechnology is also fighting its way in medicine, offering some exciting possibilities which few years ago are only imagined.

Devices operating on nanometer length scale always provide new challenges, especially regarding their thermo-mechanical properties, and re- 


\section{A. Sellitto, P. Rogolino, I. Carlomagno}

searchers face great challenges in thermal management and analysis under the extreme conditions. In fact, it is well-known that the heat-transfer process in nanosystems significantly differs from that in macrosystems [1-5]. The consequent inapplicability of the classical Fourier law in practical applications to well-describe heat transport at nanoscale has led to several generalizations of it [6-14]. Leaving untouched the differences between all theoretical approaches one can find in literature [15], it is possible to claim that each of them provides a comprehension of heat-transfer mechanism at nanoscale which is almost satisfactory. However, there is still a lack of understanding of that processes.

Among the different theoretical approaches describing heat transport beyond the Fourier law, the thermomass (TM) theory [16-18] represents a recent proposal which is growing in interest. In this theory, whose thermodynamic compatibility with second law has been analyzed in Ref. [19] within the framework of continuum thermodynamics, the following nonlinear evolution equation for the heat flux $\mathbf{q}$ is derived:

$$
\tau_{\mathrm{tm}} \frac{\partial \mathbf{q}}{\partial t}-c_{v} \ell \frac{\partial T}{\partial t} \mathbf{q}+\ell \nabla \mathbf{q} \cdot \mathbf{q}+\lambda\left(1-q^{2} b^{2}\right) \nabla T+\mathbf{q}=\mathbf{0}
$$

wherein

$$
\begin{aligned}
\ell & =\frac{\lambda \rho}{2 \gamma c_{v}\left(c_{v} T\right)^{2}} \\
b^{2} & =\frac{\rho}{2 \gamma\left(c_{v} T\right)^{3}}
\end{aligned}
$$

with $T$ being the temperature, $\tau_{\mathrm{tm}}$ a suitable relaxation time [16,19], $c_{v}$ the volumetric specific heat at constant volume, $\lambda$ the thermal conductivity, $\rho$ the mass density, and $\gamma$ the Grüneisen constant. In the evolution equation (1) the nonlinear terms are introduced by the parameters in Eqs. (2) which, indeed, do not have a precise physical meaning. However, they turn out well-known quantities when coupled with the heat flux. In fact, $\ell \mathbf{q}$ in Eq. (1) denotes the characteristic length vector of heat conduction $[17,19]$. It conceptually differs from the mean-free path of thermons, and characterizes the strength of the non-Fourier effects introduced by Eq. (1) $[17,19]$. Moreover, the quantity $q^{2} b^{2}$ denotes a dimensionless number which is also called thermal Mach number of the drift velocity relative to the thermalwave speed in the heat-carrier collection.

The TM theory can be viewed as an interesting example of the massenergy relation in thermal science. In particular, it reveals a dual nature of heat, which behaves like energy during its transformation into other forms of energy, and like mass during its transfer from the hotter bodies to the colder 


\section{Heat-pulse propagation in thermomass theory}

ones. From a microscopic point of view, the TM of an individual particle (atoms, molecules, etc.) is defined as $\hat{\mathrm{A}}$ "thermon $\hat{\mathrm{A}}$ ". All the thermons in a system form thermon gas, and the heat conduction of solids is actually the direct flow of thermon gas under its pressure gradient [20].

This theory has been successfully already used in Refs. [17,21] to describe the behavior of the effective thermal conductivity in nanomaterials, which is a very hot topic in practical applications. Indeed, besides incorporating information on the characteristic length of the system, the peculiarity of Eq. (1) is also to cope with relaxational and nonlinear effects, which are of fundamental importance for the functionality of nanosystems. Indeed, it is not our present purpose to further examine this theory in an exhaustive way, but only to point out a significant and illustrative aspect of the consequences of Eq. (1). In particular, starting from Eq. (1), in the present paper we study how thermal waves are predicted to propagate by the TM theory in nonequilibrium steady states characterized by a non-vanishing temperature gradient, or heat flux, and what may be the possible implications of those predictions in practical applications.

The structure of the paper is the following. In Sec. 2 we briefly calculate the speed of propagation of thermal pulses in TM theory. In Sec. 3 we analyze the consequences of the temperature dependence of the propagation speeds on the shape of a heat pulse. Therein we also compare the predictions of the TM theory with those arising from a different theoretical proposal. In Sec. 4 the main results obtained in the paper are summarized and discussed.

\section{Heat waves in nonequilibrium steady states}

In the classical theory, wherein heat transport is described by the Fourier law, thermal signals obey to a parabolic partial differential equation [15]. This implies that they paradoxically propagate with an infinite speed, namely, the influence of a thermal signal is immediately felt throughout the whole system. That equation, instead, becomes hyperbolic whenever relaxational effects are incorporated. This means that the aforementioned speed becomes finite, in agreement both with experimental observations, and with kinetic theory $[4,22-26]$.

Therefore, from the theoretical point of view, the search for a generalization of the Fourier law is strictly related to the problem of finding a suitable equation leading to a hyperbolic partial differential equation for the heat waves. From the experimental point of view, instead, that search began when the second sound and the ballistic phonon propagation in some dielectric solids at low temperature were discovered [27]. Such analyses are of great interest in solid-state physics because they provide useful and rel- 


\section{A. Sellitto, P. Rogolino, I. Carlomagno}

evant information on phonon scattering process [28].

A great part of the works dealing with heat-waves propagation principally focus their own attention on the analysis of the consequences of the dynamical behavior of the generalized heat-transport equation. This is a natural consequence of the very important role played by relaxational terms in high-frequencies nonequilibrium situations [28]. However, the same attention should be also put on nonlinear terms, if they are taken into account in the theoretical proposal.

Therefore, along with these observations, in the present section we briefly analyze the consequences of nonlinear terms in Eq. (1) on the heatwaves propagation. To this end, we consider a rigid solid which can be locally characterized by the internal energy per unit volume $e$ and the heat flux vector $\mathbf{q}$ [4]. The energy balance law in this case reduces to

$$
\frac{\partial e}{\partial t}=-\nabla \cdot \mathbf{q}
$$

By means of the constitutive relation $e=c_{v} T$, and assuming that the specific heat per unit volume $c_{v}$ is a constant material function ${ }^{\mathrm{a}}$, in the one-dimensional (1D) case the coupling of Eqs. (1) and (3) gets

$$
\tau_{\mathrm{tm}} \frac{\partial q}{\partial t}+2 \ell q \frac{\partial q}{\partial x}+\lambda \xi \frac{\partial T}{\partial x}+q=0
$$

wherein

$$
\xi=\left(1-q^{2} b^{2}\right)
$$

and $x$ is the longitudinal cartesian coordinate.

Referring to the classical procedure for the analysis of waves propagation $[29,30]$, let us consider a smooth surface of equation $\varphi(x ; t)=0$ propagating through the body. We assume that both the temperature $T$, and the heat flux $q$ are continuous across that surface, but their first-order derivatives suffer finite discontinuities defined by

$$
\delta=\left(\frac{\partial}{\partial \varphi}\right)_{\varphi=0^{+}}-\left(\frac{\partial}{\partial \varphi}\right)_{\varphi=0^{-}}
$$

Making use of the standard transformations

$$
\frac{\partial}{\partial t} \rightarrow-U \delta, \quad \frac{\partial}{\partial x} \rightarrow \delta
$$

\footnotetext{
${ }^{a}$ This mathematical assumption, which allows us to derive very simple and manageable relations, lays on the physical observation that in several occasions the specific heat capacity shows non-negligible variations only in very wide temperature range.
} 


\section{Heat-pulse propagation in thermomass theory}

with $U$ being the wave speed, from Eqs. (3) and (4) the following system of two homogeneous algebraic relations for the discontinuities $\delta T$ and $\delta q$ arises

$$
\left\{\begin{array}{l}
-U c_{v} \delta T+\delta q=0 \\
\lambda \xi \delta T+\left(2 \ell q_{0}-\tau_{\mathrm{tm}} U\right) \delta q=0
\end{array}\right.
$$

wherein $q_{0}$ means the steady-state heat-flux value. The homogeneous linear system (8) has nontrivial solutions if, and only if, the following relation holds:

$$
c_{v} \tau_{\mathrm{tm}} U^{2}-2 \ell c_{v} q_{0} U-\lambda \xi=0
$$

which implies that the characteristic velocities are

$$
U^{ \pm}=U_{0}\left(\sqrt{\Phi^{2}+\xi} \pm \Phi\right)
$$

with the superscript + indicating the wave traveling in the positive direction (namely, in the same direction of the heat flux), and the superscript - indicating the wave propagating in the negative direction (i.e., the opposite direction with respect to the heat flux). Moreover in Eqs. (10) $U_{0}=\sqrt{\lambda /\left(\tau c_{v}\right)}$ is the speed of propagation of thermal waves in an equilibrium reference state, and

$$
\Phi=\ell q_{0} \sqrt{\frac{c_{v}}{\lambda \alpha \tau}}
$$

with $\alpha=\tau_{\text {tm }} / \tau$, being $\tau$ the relaxation time of resistive interactions between phonons. We explicitly observe that $\tau_{\mathrm{tm}}$ in Eq. (1) means the lagging time from the temperature gradient to the corresponding heat flux in TM theory, whereas $\tau$ means the lagging time from the thermal nonequilibrium to the equilibrium state in the Maxwell-Cattaneo theory [15]. According with these observations, the nondimensional parameter $\alpha$ above may allow to compare the thermal response in the transient of both theories. As it has been observed in Ref. [19], in some cases the two relaxation times may have orders of magnitude which are sensibly different. In particular, in a silicon sample the smaller the temperature, the bigger $\tau_{\mathrm{tm}}$ than $\tau$.

From Eqs. (10) it follows that a heat wave, moving in the same direction of the average heat flow, will travel with a velocity which differs from that of the wave propagating in the opposite direction. In particular, due to the positive values of $\Phi$ arising from Eq. (11), we may conclude that the TM theory predicts that $U^{+}>U^{-}$. It is worth noticing that the absolute value of the difference in the wave speeds, i.e., $\left|U^{+}-U^{-}\right|$represents 


\section{A. Sellitto, P. Rogolino, I. Carlomagno}

a thermodynamic prediction of a relation between the speeds of thermal pulses in equilibrium (which gives information on the relaxation time) and the speeds of thermal pulses under a heat flux. In the absence of nonlinear terms in Eq. (1), instead, $\Phi=0$ and $\xi=1$ in Eqs. (10), and no difference in the wave speeds would be recovered.

Referring the reader to Sec. 3.2 for a comparison with a different theoretical proposal of the heat-transport equation, here we only observe that in phonon hydrodynamics it is usually recovered that $U^{-}>U^{+}[4,9,22]$. Moreover, in Sec. 4 we also comment that this result, which seems to be specific of the TM theory, can be used to test the validity of the TM theory in practical applications.

In closing the present section, we finally observe that the temperature dependencies of $\xi$ and $\Phi$ in Eqs. (5) and (11), respectively, point out that the propagation speeds in Eqs. (10) are temperature dependent. In the next section we will analyze the consequences of this dependence.

\section{Focusing of an energy pulse}

In previous section we observed that heat waves, propagating through a 1D system, in the TM theory are predicted to move with a finite speed, the value of which is given by Eqs. (10). Due to the nonlinear terms in Eq. (1), that value varies depending of whether the wave is moving towards the heat flux, or in the opposite direction. We further observed that the nonlinear terms are also the only responsible of the temperature dependence of Eqs. (10).

The temperature dependence of that speed may have interesting consequences in practical applications. For example, consider a rectangular energy pulse traveling through a system which is in a nonequilibrium situation due to the presence of a temperature gradient applied between its two ends. Previous observations allow us to argue that the two boundaries of the energy pulse should move with slightly different speeds. To analyze the possible consequences of these speed differences, we introduce the following (finite) pulse width

$$
\Delta x^{ \pm}=x_{\mathrm{f}}^{ \pm}-x_{\mathrm{r}}^{ \pm}
$$

with $x_{\mathrm{f}}(T)$ being the position of the front edge, and $x_{\mathrm{r}}(T)$ the position of the rear edge (the superscript + still standing for the positive direction of propagation, and the superscript - for the negative direction).

Then, it is easy to see that the change of $\Delta x^{ \pm}$as a function of the 


\section{Heat-pulse propagation in thermomass theory}

position along the system during the propagation of the pulse, reads

$$
\begin{aligned}
& \frac{d}{d x}\left(\Delta x^{ \pm}\right)=\left[\frac{d}{d t}\left(\Delta x^{ \pm}\right)\right] \frac{d t}{d x}=\frac{1}{U^{ \pm}}\left(\frac{d x_{\mathrm{f}}^{ \pm}}{d t}-\frac{d x_{\mathrm{r}}^{ \pm}}{d t}\right)=\frac{U^{ \pm}\left(T_{\mathrm{f}}\right)-U^{ \pm}\left(T_{\mathrm{r}}\right)}{U^{ \pm}}= \\
& (13) \quad \frac{1}{U^{ \pm}}\left(\frac{d U^{ \pm}}{d T}\right)\left(\frac{d T}{d x}\right) d x \approx\left(\frac{d \ln U^{ \pm}}{d T}\right)\left(\frac{d T}{d x}\right) \Delta x^{ \pm}
\end{aligned}
$$

By straightforward calculations it is also possible to observe that the coupling of Eqs. (10) and (13) turns out that the relative variation rate of the width of a traveling heat pulse along the system, predicted by the TM theory, is

$$
\begin{aligned}
\Delta X^{ \pm}= & \frac{1}{\Delta x^{ \pm}}\left[\frac{d}{d x}\left(\Delta x^{ \pm}\right)\right]= \\
& \frac{1}{T\left(\sqrt{\Phi^{2}+\xi} \pm \Phi\right)}\left[\frac{3(1-\xi)-4 \Phi^{2}}{2 \sqrt{\Phi^{2}+\xi}} \mp 2 \Phi\right]\left(\frac{d T}{d x}\right)
\end{aligned}
$$

From a practical point of view, it is also interesting to analyze the evolution of the pulse amplitude $\theta^{ \pm}$. To this end, if we suppose that the total internal energy of the heat pulse $I^{ \pm}=c_{v} \theta^{ \pm} \Delta x^{ \pm}$is constant ${ }^{\mathrm{b}}$, then the relative variation rate of the pulse amplitude is

$$
\frac{d I^{ \pm}}{d x}=0 \Rightarrow \Delta \Theta^{ \pm}=\frac{1}{\theta^{ \pm}}\left(\frac{d \theta^{ \pm}}{d x}\right)=-\frac{1}{\Delta x^{ \pm}}\left[\frac{d}{d x}\left(\Delta x^{ \pm}\right)\right]=-\Delta X^{ \pm}
$$

which implies that if the pulse width shrinks (i.e., if in Eq. (14) $d\left(\Delta x^{ \pm}\right) / d x<0$ ), then the pulse amplitude raises up (i.e., in Eq. (15) $d \theta^{ \pm} / d x>0$ ), whereas if the pulse width enlarges (i.e., if in Eq. (14) $d\left(\Delta x^{ \pm}\right) / d x>0$ ), then the pulse amplitude gets squashed (i.e., in Eq. (15) $\left.d \theta^{ \pm} / d x<0\right)$.

\subsection{Application to a silicon nanowire}

In order to apply previous results to a concrete situation, consider a nanowire of longitudinal length $L$ (much larger with respect to the radius of the transversal section in such a way that it may be assumed as a $1 \mathrm{D}$ system) in steady states, the hotter side of which is kept at the constant

\footnotetext{
${ }^{b}$ In practical applications, the amount of the heat contained in the pulse is not always the same during the propagation. In fact, due to the diffusion effects, that amount is distributed to the regions of lower temperature. However, if the characteristic time of diffusion effects is larger than that of the pulse propagation, in principle it is possible to assume that $I^{ \pm}$is constant.
} 


\section{A. Sellitto, P. Rogolino, I. Carlomagno}

temperature $T_{h}$, whereas its colder side is at the constant temperature $T_{c}$. In particular, just for the sake of computation, assume that the nanowire is made by silicon, $L=10^{-4} \mathrm{~m}, T_{h}=330 \mathrm{~K}$, and $T_{c}=270 \mathrm{~K}$. The Grüneisen constant of silicon is $\gamma=1.5$ [31].

For simplicity, we also assume that across the two ends of the nanowire a linear temperature gradient of the form

$$
T(x)=-\frac{x}{L}\left(T_{h}-T_{c}\right)+T_{h}
$$

is applied. This implies that the system at hand can be supposed at the average temperature $\bar{T}=300 \mathrm{~K}$. The thermal conductivity of silicon at the room temperature is $\lambda=148 \mathrm{~W} \mathrm{~m}^{-1} \mathrm{~K}^{-1}$, whereas the specific heat at constant volume per unit volume may be obtained by using the usual Debye expression, namely, $c_{v}=\left(12 \pi^{4} / 5\right)\left(\bar{T} / T_{B}\right)^{3}\left(R \rho_{\mathrm{Si}} / M_{\mathrm{Si}}\right)$, with $T_{B}=645 \mathrm{~K}$ as the Debye temperature for silicon, $R=8.31 \mathrm{JK}^{-1} \mathrm{~mol}^{-1}$ as the gas constant, $M_{\mathrm{Si}}=28 \times 10^{-3} \mathrm{~kg} \mathrm{~mol}^{-1}$ as the silicon molar mass, and $\rho_{\mathrm{Si}}=2.33 \times 10^{3} \mathrm{~kg} \mathrm{~m}^{-3}$ as the mass density of silicon.

Along with previous results, the TM theory predicts that a rectangular energy pulse traveling longwise the system (see Fig. 1 for a qualitative sketch) will change its shape both whether it moves in the same direction $(+)$ of the average heat pulse $q_{0}$, and in the opposite direction $(-)$.

By using Eqs. (14) and (15), in Fig. 2 we plot the relative variation rate of the pulse amplitude. In obtaining the value of $\alpha$ to use in Eq. (11) we calculated the relaxation time in the TM theory as $\tau_{\mathrm{tm}}=\lambda \rho /\left(2 \gamma c_{v}^{2} \bar{T}\right)$, and we roughly estimated the relaxation time of resistive interactions between phonons as $\tau=\ell_{p} / \bar{c}$, with $\bar{c}=8.34 \times 10^{3} \mathrm{~m} \mathrm{~s}^{-1}$ being the phonon speed, and $\ell_{p}=40.3 \times 10^{-9} \mathrm{~m}$ being the phonon mean-free path at the room temperature. Moreover, we calculated the average heat flux flowing throughout our system as $q_{0}=\lambda\left(T_{h}-T_{c}\right) / L$.

As one can clearly see, the pulse traveling in the same direction of $q_{0}$ is characterized by a positive value of $\Delta \Theta^{+}$everywhere. Along with previous observations, in this case we may conclude that the pulse shrinks, growing this way its temperature. In practical applications this may cause some problems if the system cannot stand at high temperatures. In fact, in this case, the propagating pulse may lead to the consequence that some points of the nanowire will stay at a temperature which is higher than the melting temperature threshold.

The pulse traveling in the opposite direction of $q_{0}$, instead, is always characterized by a negative value of $\Delta \Theta^{-}$. This means that the pulse enlarges during its motion with the consequent reduction of the temperature. This may be also a problem in practical applications if one is trying, for 


\section{Heat-pulse propagation in thermomass theory}
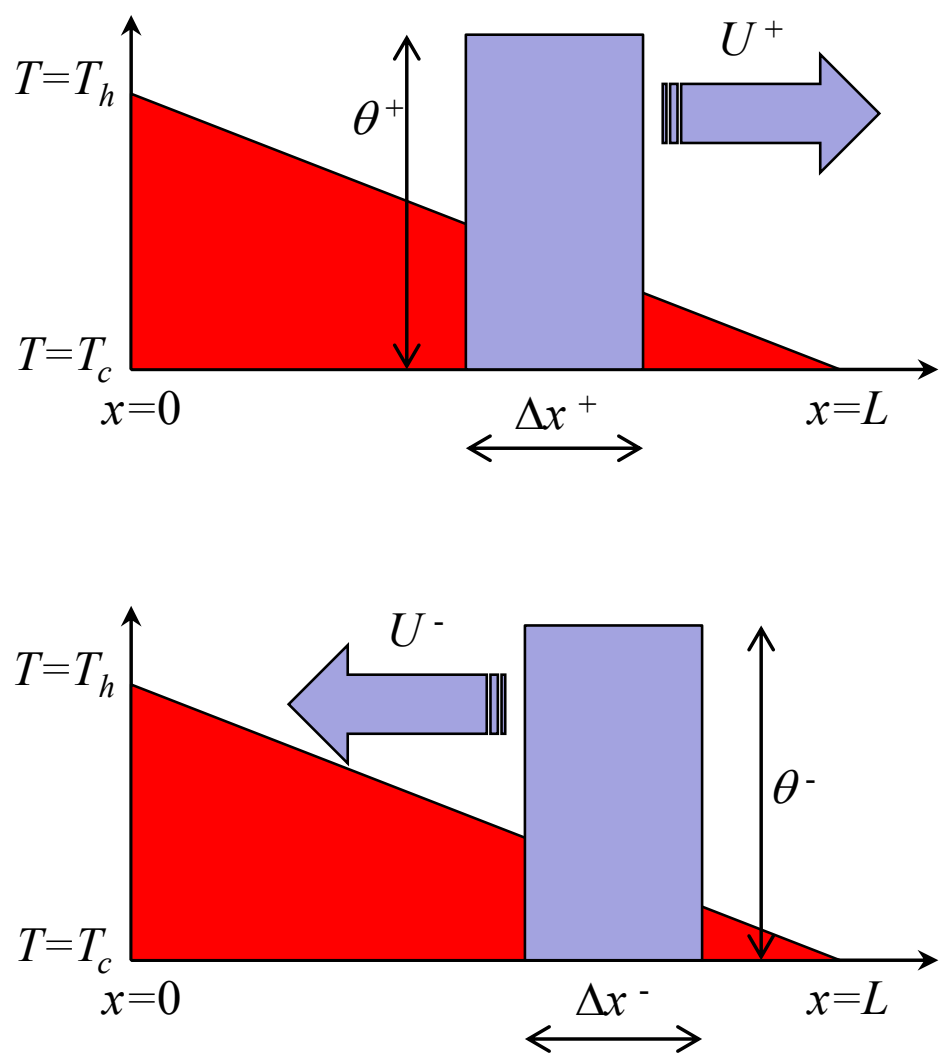

Figure 1. Figure shows a rectangular energy pulse moving throughout a nanowire when a linear temperature gradient between the two ends of which is applied. The end at $x=0$ is held at the hot temperature $T_{h}$, and the end at $x=L$ is held at the cold temperature $T_{c}$. When the energy pulse travels from left to right in figure (i.e., from $x=0$ to $x=L$ ), it moves in the same direction $(+)$ of the average heat flux $q_{0}$, and its speed is $U^{+}$. When the energy pulse, instead, travels from right to left in figure (i.e., from $x=L$ to $x=0$ ), it moves in the opposite direction (-) of the average heat flux $q_{0}$, and its speed is $U^{-}$.

example, to send information along the system by means of heat pulses. If the temperature of the pulses becomes too small, in fact, it will not be detected.

Although a more refined analysis, (for example, a detailed microscopic analysis of phonon focusing based on elastic properties of crystals), is desirable, previous results allow to infer that nonlinear effects may play a crucial role in some situations, and they should be never neglected. 


\section{A. Sellitto, P. Rogolino, I. Carlomagno}
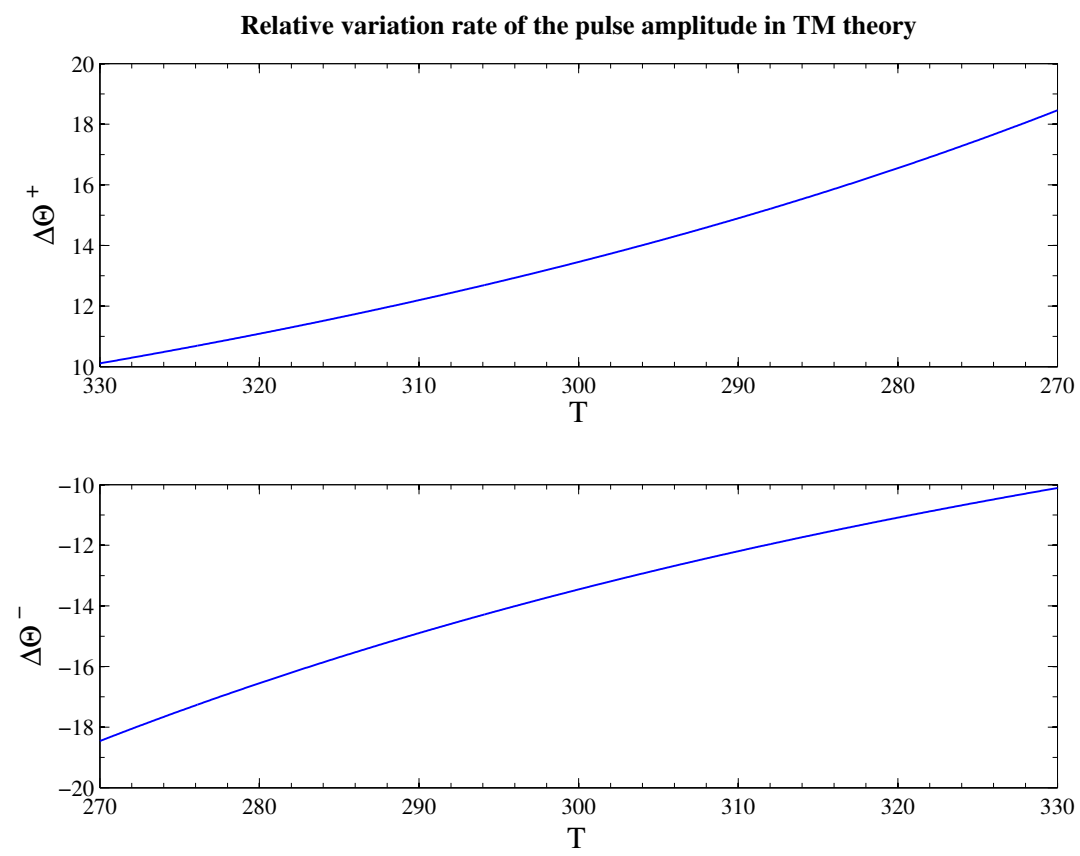

Figure 2. Behavior of the relative variation rate of the pulse amplitude in a silicon nanowire versus the temperature: theoretical results predicted by the coupling of Eqs. (14) and (15). The superscript + denotes the aforementioned behavior for a pulse traveling in the same direction of the average heat flux $q_{0}$, i.e., a pulse which moves from the hotter end to the colder one. The superscript -, instead, denotes the behavior of a pulse traveling in the opposite direction of the average heat flux $q_{0}$, i.e., a pulse moving from the colder end to the hotter one.

\subsection{Comparison with a different formalism}

With the rapid development of femtosecond lasers and micro/nano processing techniques, the meaning of temperature in nonequilibrium situations is becoming a very acute problem, because of the relatively small number of particles involved and the influence of fast variations. In order to comply with these situations, in recent papers $[9,32,33]$ the concept of dynamical temperature $\beta$ has been introduced. By design, $\beta$ coincides with the absolute temperature $T$ at equilibrium, otherwise $\beta$ follows $T$ with a certain delay which is controlled by a suitable relaxation time $[32,33]$. The dynamical temperature, which can be fundamentally regarded as an additional degree of freedom, is related to internal forcing acting on the heat carriers which manifest themselves at time scales of the order of its characteristic relaxation time. 


\section{Heat-pulse propagation in thermomass theory}

Starting from the idea that the heat flux $\mathbf{q}$ is proportional to the gradient of $\beta[9,32,33]$, namely, $\mathbf{q}=-\lambda \nabla \beta$, in Ref. [9] the authors derived the following nonlinear Maxwel-Cattaneo type (nMC) evolution equation for the heat flux

$$
\tau \frac{\partial \mathbf{q}}{\partial t}+\mathbf{q}-\frac{2 \tau}{T c_{v}} \mathbf{q} \cdot \nabla \mathbf{q}+\lambda \nabla T=\mathbf{0}
$$

which leads to the following speeds for heat waves [9]

$$
U^{ \pm}=U_{0}\left(\sqrt{\phi^{2}+1} \mp \phi\right)
$$

wherein $\phi=q_{0} /\left(T c_{v} U_{0}\right)$. The positive defined character of $\phi$ in Eqs. (18) turns out that the theoretical proposal (17) predicts that $U^{-}>U^{+}$, in agreement with usual results of phonon hydrodynamics [4,9,22], but in contrast with the predictions of TM theory derived in Sec. 2. However, the temperature dependence of $\phi$ in Eqs. (18) points out that also in this formalism both propagation speeds are temperature dependent, implying that the two boundaries of a energy pulse move with slightly different speeds.

We note that the consequences of accounting for Eq. (17) on the shape of an energy pulse propagating along a system in nonequilibrium situations have been analyzed in deep in Ref. [25] in the more general case of temperature-dependent material functions. Here we only consider the simpler situation in which the different material functions do not depend on the temperature (or, equivalently, all of them show negligible variations owing to the applied temperature gradient) in order to compare the predictions of the TM theory with those of the nMC theory.

By direct calculations it is easy matter to see that in this case the relative variation rates of the pulse width read

$$
\Delta X^{ \pm}=\frac{ \pm \phi}{T\left(\sqrt{\phi^{2}+1} \mp \phi\right)}\left[1 \mp \frac{\phi}{\sqrt{\phi^{2}+1}}\right]\left(\frac{d T}{d x}\right)
$$

Still assuming that our system is submitted to the linear temperature gradient in Eq. (16), with $T_{h}=330 \mathrm{~K}$ and $T_{c}=270 \mathrm{~K}$, in Fig. 3 we plot the relative variation rate of the pulse amplitude predicted by the $\mathrm{nMC}$ equation (17). The results plotted therein allow to see that, in contrast with the prediction of the TM theory, in this different formalism a heat pulse propagating in the same direction of the average heat squashes and its temperature decreases, whereas a heat pulse propagating in the opposite direction shrinks and increases its temperature. 
A. Sellitto, P. Rogolino, I. Carlomagno
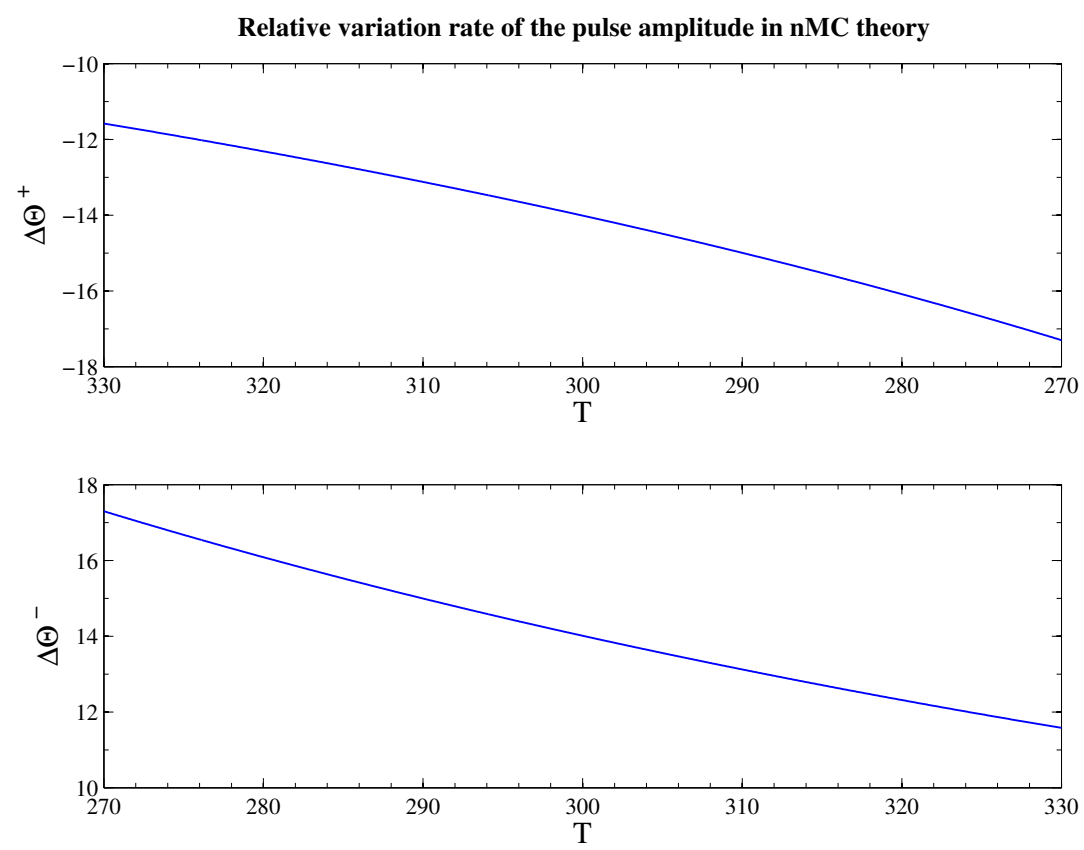

Figure 3. Behavior of the relative variation rate of the pulse amplitude in a silicon nanowire versus the temperature: theoretical results predicted by the coupling of Eqs. (15) and (19). The superscript + denotes the aforementioned behavior for a pulse traveling in the same direction of the average heat flux $q_{0}$, i.e., a pulse which moves from the hotter end to the colder one. The superscript -, instead, denotes the behavior of a pulse traveling in the opposite direction of the average heat flux $q_{0}$, i.e., a pulse moving from the colder end to the hotter one.

\section{Conclusions}

In the present paper we analyzed the consequences predicted by the TM theory [16-18] on the speed of propagation of heat waves. In the special case of a silicon nanowire submitted to a linear temperature gradient, we pointed out the very important role that nonlinear effects may have in practical applications. In more detail, in Sec. 3 we observed that those effects may be responsible of changes in the shape of a heat pulse traveling through a nanowire.

It is worth noticing that in Eq. (1) the nonlinear effects are accounted by the parameters $\ell$ and $b^{2}$ in Eqs. (2). Indeed, for the case analyzed in the present paper, the nonlinear effects introduced by the former parameter are prevalent with respect to those introduced by the latter parameter, which can be also related to the concept of flux limiters $[4,34,35]$. Along with this 


\section{Heat-pulse propagation in thermomass theory}

observation, here we also compared those results with the predictions of the different theory proposed in Ref. [9], which introduces in Eq. (17) the nonlinear effects by means of a term which is very similar (but not equal) to $\ell$.

Although it is evident by now the importance of accounting for nonlinear effects in the heat-transport equation at nanoscale, we note that the different theories should introduce them in such a way that the mathematical form of the different characteristic equations remains always the same. Therefore, the present analysis, which in a first rough look may appear only as an academic motivation, becomes acute if one observes that the growing importance of nanostructures fostered the interest in generalized heat-transport equations incorporating memory, nonlocal, and nonlinear effects. Owing this, in literature different theories and/or models leading to a generalization of the classical Fourier law may be found [15,27]. Although from the theoretical point of view one has to admit that some of them are very refined, while others show some weak points, in general it is not so simple to claim which is the best (or, alternatively said, the right) proposal. To this end, a suitable way of testing the validity of a given model with respect to another one may be to find practical (and simple to be reproduced) applications wherein different theories clearly predict different results. This is, for example, just the case of the propagation of energy pulse in a nanowire we analyzed in the present paper, which could be used to check the validity of Eq. (1) with respect to Eq. (17). In fact, Eq. (1) predicts that an energy pulse propagating through a silicon nanowire shrinks (and increases in temperature) when it moves from the hot side to the cold one, whereas that pulse enlarges (and decreases its temperature) when it passes from the cold side to the hot one. The theoretical model introduced by Eq. (17), instead, exactly predicts the opposite behaviors.

Indeed, in closing the present paper let us observe that differences between the TM theory and other modern thermodynamic theories, as for example extended thermodynamics [15], also rest on the difference $U^{-}-U^{+}$, which in the former theory is negative, and in the latter is always positive. In that respect, a comparison with experimental data should have been welcome to decide between those opposite results, too. For example, a possible system wherein at high frequencies the difference $\left|U^{-}-U^{+}\right| \neq 0$ is sketched in Fig. 4. However, in the book by Jou et al [4] (see therein Part III - Selected Applications) it is possible to find several examples of practical applications in which one can measure two different speeds for propagating thermal pulses. 

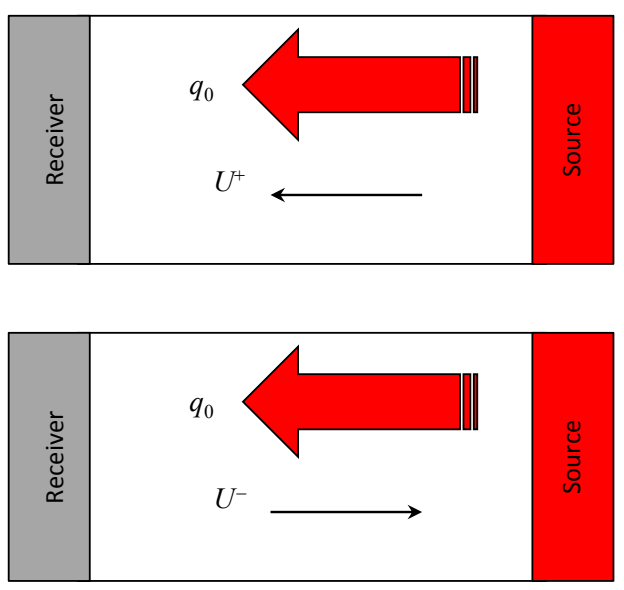

Figure 4. In the right-hand side of the system a source of the heat pulse (for example a faster heater) is directly connected with a heat-pulse receiver (for example a superconducting bolometer). The system is in a nonequilibrium situation when an average heat flux $\mathbf{q}_{0}$ is flowing through it.

\section{Acknowledgements}

The authors acknowledge the financial support of the Italian Gruppo Nazionale per la Fisica Matematica - GNFM. 


\section{Heat-pulse propagation in thermomass theory}

\section{REFERENCES}

1. T. L. Hill, Thermodynamics of Small Systems. New York: Dover, 1994.

2. D. Y. Tzou, Macro to micro-scale heat transfer. The lagging behaviour. New York: Taylor and Francis, 1997.

3. Z. M. Zhang, Nano/Microscale heat transfer. New York: McGraw-Hill, 2007.

4. D. Jou, J. Casas-Vázquez, and G. Lebon, Extended Irreversible Thermodynamics. Berlin: Springer, fourth revised ed., 2010.

5. S. Volz (ed.), Thermal Nanosystems and Nanomaterials (Topics in Applied Physics). Berlin: Springer, 2010.

6. R. A. Guyer and J. A. Krumhansl, "Solution of the linearized phonon Boltzmann equation," Phys. Rev., vol. 148, pp. 766-778, 1966.

7. G. Chen, "Ballistic-diffusive heat-conduction equations," Phys. Rev. Lett., vol. 86, pp. 2297-2300, 2001.

8. M. Grmela, G. Lebon, P. C. Dauby, and M. Bousmina, "Ballisticdiffusive heat conduction at nanoscale: GENERIC approach," Phys. Lett. A, vol. 339, pp. 237-245, 2005.

9. V. A. Cimmelli, A. Sellitto, and D. Jou, "Nonlocal effects and second sound in a nonequilibrium steady state," Phys. Rev. B, vol. 79, p. 014303 (13 pages), 2009.

10. D. Y. Tzou and Z.-Y. Guo, "Nonlocal behavior in thermal lagging," Int. J. Thermal Sci., vol. 49, pp. 1133-1137, 2010.

11. D. Y. Tzou, "Nonlocal behavior in phonon transport," Int. J. Heat Mass Transfer, vol. 54, pp. 475-481, 2011.

12. G. Lebon, H. Machrafi, M. Grmela, and C. Dubois, "An extended thermodynamic model of transient heat conduction at sub-continuum scales," Proc. R. Soc. A, vol. 467, pp. 3241-3256, 2011.

13. P. Ván and T. Fülöp, "Universality in heat conduction theory: weakly nonlocal thermodynamics," Ann. Phys., vol. 524, pp. 470-478, 2012.

14. G. Lebon, "Heat conduction at micro and nanoscales: A review through the prism of Extended Irreversible Thermodynamics," J. Non-Equilib. Thermodyn., vol. 39, pp. 35-59, 2014.

15. V. A. Cimmelli, D. Jou, T. Ruggeri, and P. Ván, "Entropy Principle and Recent Results in Non-Equilibrium Theories," Entropy, vol. 16, pp. 1756-1807, 2014.

16. Y. Dong, B.-Y. Cao, and Z.-Y. Guo, "Generalized heat conduction laws 


\section{A. Sellitto, P. Rogolino, I. Carlomagno}

based on thermomass theory and phonon hydrodynamics," J. Appl. Phys., vol. 110, p. 063504 (6 pages), 2011.

17. M. Wang, N. Yang, and Z.-Y. Guo, "Non-Fourier heat conductions in nanomaterials," J. Appl. Phys., vol. 110, p. 064310 (7 pages), 2011.

18. Y. Dong, B.-Y. Cao, and Z.-Y. Guo, "General expression for entropy production in transport processes based on the thermomass model," Phys. Rev. E, vol. 85, p. 061107 (8 pages), 2012.

19. A. Sellitto and V. A. Cimmelli, "A continuum approach to thermomass theory," J. Heat Trans. - T. ASME, vol. 134, p. 112402 (8 pages), 2012.

20. B.-Y. Cao and Z.-Y. Guo, "Equation of motion of a phonon gas and non-Fourier heat conduction," J. Appl. Phys., vol. 102, p. 053503 (6 pages), 2007.

21. Y. Dong, B.-Y. Cao, and Z.-Y. Guo, "Size dependent thermal conductivity of Si nanosystems based on phonon gas dynamics," Physica E, vol. 56, pp. 256-262, 2014.

22. I. Müller and T. Ruggeri, Rational Extended Thermodynamics. Berlin: Springer-Verlag, 1998.

23. J. Wang and J.-S. Wang, "Carbon nanotube thermal transport: ballistic to diffusive," Appl. Phys. Lett., vol. 88, p. 111909 (3 pages), 2006.

24. M. Fujii, X. Zhang, H. Xie, H. Ago, K. Takahashi, T. Ikuta, H. Abe, and T. Shimizu, "Measuring the thermal conductivity of a single carbon nanotube," Phys. Rev. Lett., vol. 95, p. 065502 (4 pages), 2005.

25. D. Jou and A. Sellitto, "Focusing of heat pulses along nonequilibrium nanowires," Phys. Lett. A, vol. 374, pp. 313-318, 2009.

26. Z.-Y. Guo and Q.-W. Hou, "Thermal wave based on the thermomass model," J. Heat Trans - T. ASME, vol. 132, p. 072403 (6 pages), 2010.

27. V. A. Cimmelli, "Different thermodynamic theories and different heat conduction laws," J. Non-Equilib. Thermodyn., vol. 34, pp. 229-333, 2009.

28. D. Jou, A. Sellitto, and F. X. Alvarez, "Heat waves and phonon-wall collisions in nanowires," Proc. R. Soc. A, vol. 467, pp. 2520-2533, 2011.

29. A. Jeffrey and T. Taniuti, Nonlinear Wave Propagation. New York: Academic, 1964.

30. B. Straughan, Heat waves. Berlin: Springer, 2011.

31. M. T. Yin and M. L. Cohen, "Theory of lattice-dynamical properties of solids: Application to Si and Ge," Phys. Rev. B, vol. 26, pp. 3259-3272, 


\section{Heat-pulse propagation in thermomass theory}

1992.

32. V. A. Cimmelli and W. Kosiński, "Nonequilibrium semi-empirical temperature in materials with thermal relaxation," Arch. Mech., vol. 43, pp. 753-767, 1991.

33. V. A. Cimmelli and K. Frischmuth, "Determination of material functions through second sound measurements in a hyperbolic heat conduction theory," Mathl. Comput. Modelling, vol. 24, pp. 19-28, 1996.

34. C. D. Levermore and G. C. Pomraning, "A flux-limited diffusion theory," Astrophys. J., vol. 248, pp. 321-334, 1981.

35. A. Sellitto and V. A. Cimmelli, "Flux Limiters in Radial Heat Transport in Silicon Nanolyers," J. Heat Trans. - T. ASME, vol. 136, p. 071301 (6 pages), 2014. 